\title{
Genome-wide identification of MYB family genes potentially related to SCW biogenesis in Korla pear fruit
}

\author{
Kuerban Tusong ${ }^{1}$, Aisajan Mamat ${ }^{1}$, Hongmei $\mathrm{Mu}^{2}$ and Juan $\mathrm{Xu}^{1}$ \\ ${ }^{1}$ Institute of Horticultural Crops, Xinjiang Academy of Agricultural Sciences, Urumqi, China \\ 2 Yantai Academy of Agricultural Sciences, Yantai, Shandong, China
}

\section{Summary}

The formation of fruits with a rough-skin is a physiological disorder in Korla pear (Pyrus sinkiangensis Yü) that is increasingly common in recent years. Compared with normal fruits, a main characteristic of such rough-skinned fruits is their greater stone cell content. These pear stone cells belong to the sclerenchyma formed by the secondary thickening of parenchyma cell walls, and MYB (myeloblastosis) family transcription factors (TFs) are known to have important functions during secondary cell wall (SCW) biosynthesis. Therefore, this work aimed to investigate MYB family genes in the pear genome and to identify SCW-related MYB family members in Korla pear fruits. A total of $193 \mathrm{PbMYBs}$, which were further divided into 11 clades, were identified in the pear genome. The gene phylogeny and a similarity and structure analysis showed that 33 PbMYBs were closely homologous to SCW-associated MYBs in Arabidopsis. Gene expression analysis revealed that 14 of them were differentially expressed during the experimental time course (prime, late, and stationary stage of stone cell differentiation), being homologs to the SCWrelated genes MYB20, MYB42, MYB52, MYB83, MYB85, and $M Y B 103$ in Arabidopsis. Expression pattern clusters indicated that for most of these $M Y B s^{\prime}$ expression levels peaked in the prime stage of stone cell differentiation, and then were gradually down-regulated in subsequent stages. Furthermore, RT-qPCR results showed that the pear homologs of AtMYB20, AtMYB42, AtMYB52, and AtMYB85 - downstream TFs of the SCW biosynthesis regulatory network - were significantly upregulated in either or both the peel and pulp of rough-skinned fruits. These results suggest these SCW-associated PbMYBs might play fundamental roles during the stone cell differentiation process in pear fruits.

Keywords

Korla pear, rough-skinned fruit, pear genome, MYB, SCW

\section{Introduction}

Transcription factors (TFs) are proteins that bind to the specific DNA sequences and can form complexes, either alone or with other proteins, to regulate gene expression by enhancing or interfering the recruitment of specific genes to RNA polymerase (Liu et al., 1999; Riechmann and Ratcliffe, 2000). TFs are located relatively upstream in signaling pathways, and first to respond signaling substances or environ-

\section{Significance of this study}

What is already known on this subject?

- The stone cells are formed by secondary thickening of parenchyma cell walls, and MYB family transcription factors (TFs) play important functions during the secondary cell wall (SCW) biosynthesis. Previous studies on pear fruit stone cells mainly focused on transcriptional regulation of some lignin synthetic genes by $M Y B S$.

\section{What are the new findings?}

- SCWs are mainly composed of cellulose, hemicellulose and lignin. Therefore, the formation of stone cells could not be fully explained merely by lignin deposition. In this work, we identified the homologs of MYB46/83, MYB20, MYB42, MYB52, MYB85 and MYB103 of Arabidopsis that regulate the SCWs synthesis. All of these MYBs were abundantly expressed in the critical stages of stone cell differentiation, as well as in the pericarp and pulp of rough-skinned fruits. The genes related to cellulose, hemicellulose, and lignin synthesis are the direct targets of these MYBs. This illuminates that the stone cell differentiation requires synergistic regulation of many genes and proteins that are involved in SCW related pathways.

What is the expected impact on horticulture?

- The skin roughening of pear fruits has been adversely affecting the fruit quality of Korla pears recently. Findings in this work may contribute to the understanding of the regulatory mechanism of this phenotype, and would help to take effective measures to reduce or eliminate this physiological disorder during pear production.

mental stimulus, and then regulate the downstream target genes (Riechmann and Ratcliffe, 2000; Zhang et al., 2018). Therefore, in plants many biological processes or cellular activities require involvement of TFs (Dubos et al., 2010; Mmadi et al., 2017).

The MYB family is one of the important TF superfamily in both animal and plant kingdom, R2R3-MYB proteins are the most predominant MYBs in plants (Martin and Paz-Ares, 1997; Dubos et al., 2010). Hitherto, the MYB family genes have been identified at genomic scale in Citrus sinensis (Hou et al., 2014), Solanum lycopersicum (Zhao et al., 2014), Pyrus bretschneideri (Cao et al., 2016a; Li et al., 2016), Brassica napus (Hajiebrahimi et al., 2017) and Prunus persica (Zhang et 
al., 2018). Furthermore, some of the members of this family have been functionally studied in plants. Their results showed that the MYBs involved in regulating a wide range of biological processes, including anthocyanin biosynthesis (Lin-Wang et al., 2010; Tuan et al., 2015), the coloration of fruit and flower (Tuan et al., 2015), flavonoid and phenylpropanoid metabolism (Medina-Puche et al., 2014), sugar signaling (Chen et al., 2017), and responses to drought and waterlogging stress (Mmadi et al., 2017). One of the most important functions of $M Y B s$ is regulating the synthesis of SCWs. The MYB4, MYB7, MYB26, MYB32, MYB46, MYB58, MYB63, MYB83 and MYB103 in Arabidopsis, and their homologs in other plants have been reported to regulate SCW synthesis (Mitsuda et al., 2005; Yang et al., 2007; McCarthy et al., 2009; Zhou et al., 2009; Hussey et al., 2013; Scully et al., 2016; Yang and Wang, 2016; Guo et al., 2017). Most of these TFs are related to the transcriptional activation of genes that regulate the synthesis of secondary wall principal components: cellulose, hemicellulose and lignin (Zhong et al., 2007; Ko et al., 2009; Zhong and Ye, 2012; Kim et al., 2013). Korla pear (Pyrus sinkiangensis Yü) is a native cultivar of the Xinjiang Autonomous Region, China. The fruit is known for its unique aroma, juicy flesh and crisp texture. The presence of stone cells is an important characteristic of the pear fruit quality. In regular fruits, the stone cells are unperceivable. However, the high stone cell content adversely affects the external and internal quality of pear fruit. The stone cells in pear fruits belong to the sclerenchyma that are formed by the secondary thickening of parenchyma cell walls (Lu et al., 2014; Cao et al., 2016b; Wang et al., 2017), mainly composed of cellulose, hemicellulose and lignin (Brahem et al., 2017; Mamat et al., 2019). In recent years, the occurrence of roughskinned fruits is one of the major factors that affect the local pear fruit industry, and the increased stone content is one of the typical characteristics of this phenotype. Therefore, this work attempts to identify the members of MYB family that are associated with the regulation of SCW synthesis in Korla pear fruits. The findings might be helpful to find the upstream signaling molecules or environmental factors that trigger the stone cell differentiation or formation of roughskinned fruits. Expected results also could provide the theoretical basis for taking effective measures to control the pericarp roughening during pear production.

\section{Materials and methods}

\section{Identification of MYB family genes in pear and Arabidopsis}

The genomic information of pear (Pyrus bretschneideri) was downloaded from the genome database of Rosaceae (https://www.rosaceae.org/), and genome of Arabidopsis thaliana was downloaded from the Arabidopsis Information Resource (TAIR) database (http://www.arabidopsis. org/). The PbMYB (MYB family genes in P. bretschneideri) and $A t M Y B$ genes (MYB family genes in $A$. thaliana) were searched against Hidden Markov Model (HMM) of MYB domain (PF00249) downloaded from Pfam database (http:// pfam.xfam.org/) with the E-value of $1 \mathrm{e}-10$. The protein sequences identified as containing MYB domain were subjected to search against Pfam database and SMART tools (http://smart.embl-heidelberg.de/) for further confirmation of presence of MYB domain. The confirmed sequences were further searched against Conserved Domains Database (CDD) (http://www.ncbi.nlm.nih.gov/cdd/) for checking the completeness of domain structure. At last, iTAK online program (http://itak.feilab.net/cgi-bin/itak/index.cgi) was used for further checking whether these TFs could be classified as MYBs.

\section{Homologs of SCW biogenesis related AtMYBs in pear}

OmicBox software (https://www.biobam.com/omicsbox/) was used to annotate the PbMYBs in different

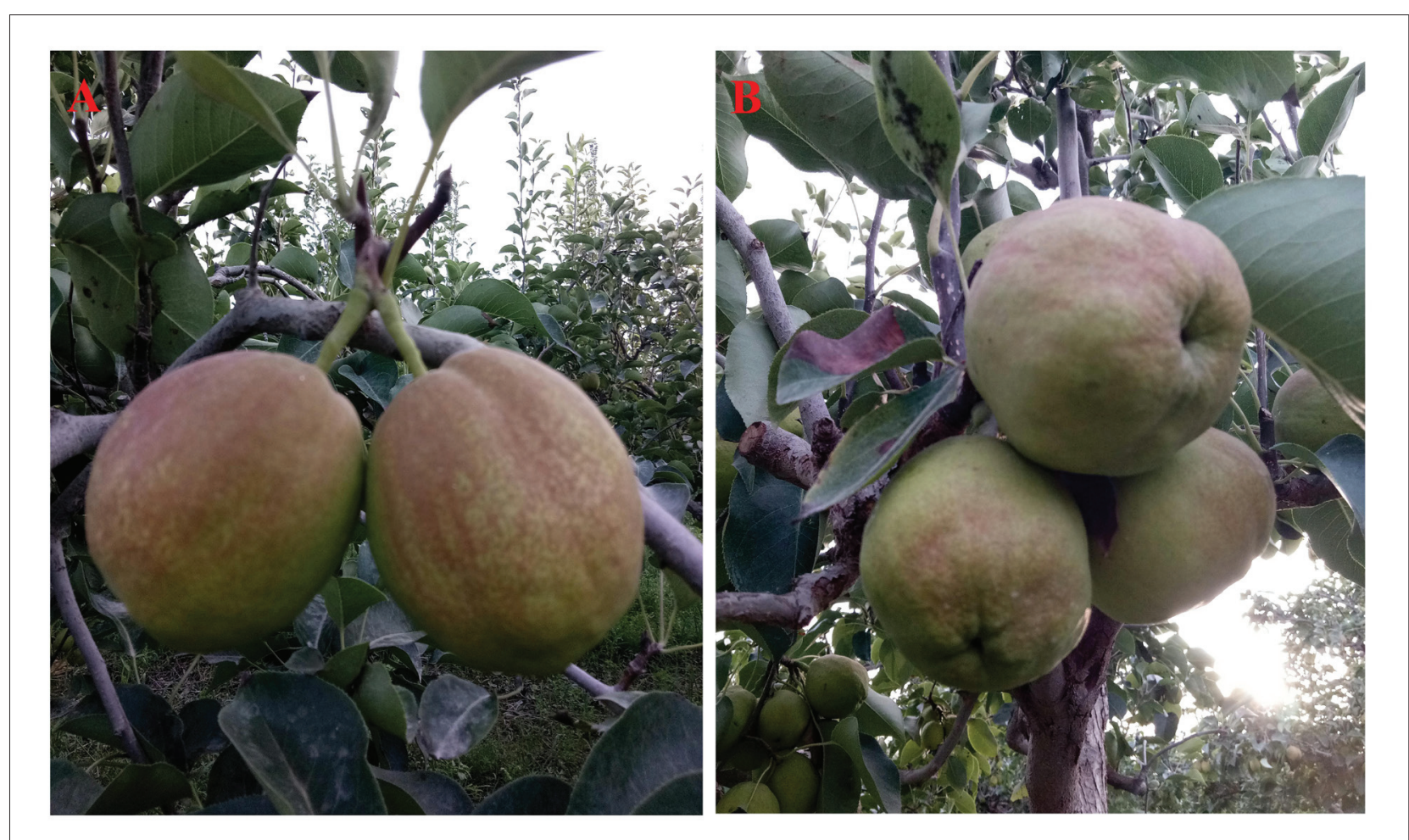

FigURE 1. Fruits of Korla pear (Pyrus sinkiangensis Yü) at maturity stage. A is the normal fruits and B is the rough-skinned fruits. 
biological presses, including SCW biosynthesis. MYB family genes associated with this process were gathered by searching publications that have been functionally verified in Arabidopsis. The sequences of PbMYBs and AtMYBs were aligned with MUSCLE program, and phylogenetic relationships were constricted by using un-rooted neighbor-joining (NJ) method of MEGA-X (Molecular Evolutionary Genetics Analysis) software. The reliability of phylogenetic tree was tested by bootstrapping method of 1,000 replicates. For identification of closely homologous sequences of SCW associated AtMYBs in pear, PbMYBs and AtMYBs were aligned by using BLAST2.6.0+ software with $E$-value $<1 \mathrm{e}-10$.

\section{Gene structure and motif analyses of the SCW associated $M Y B S$}

Genomic coordinates of different regions of corresponding $P b M Y B$ and $A t M Y B$ genes were uploaded on online tool: Gene Structure Display Server (GSDS) (http://gsds.cbi.pku. edu.cn/), and the gene structures were visualized and downloaded. Meanwhile, MEME 4.12.0 online software package (http://meme-suite.org/) was used for the prediction of motif structures. The employed parameters of MEME program were as follows: maximum number of motifs to "20", number of repetitions to "any", the width of each motif was between 6 to 50 amino acid residues.

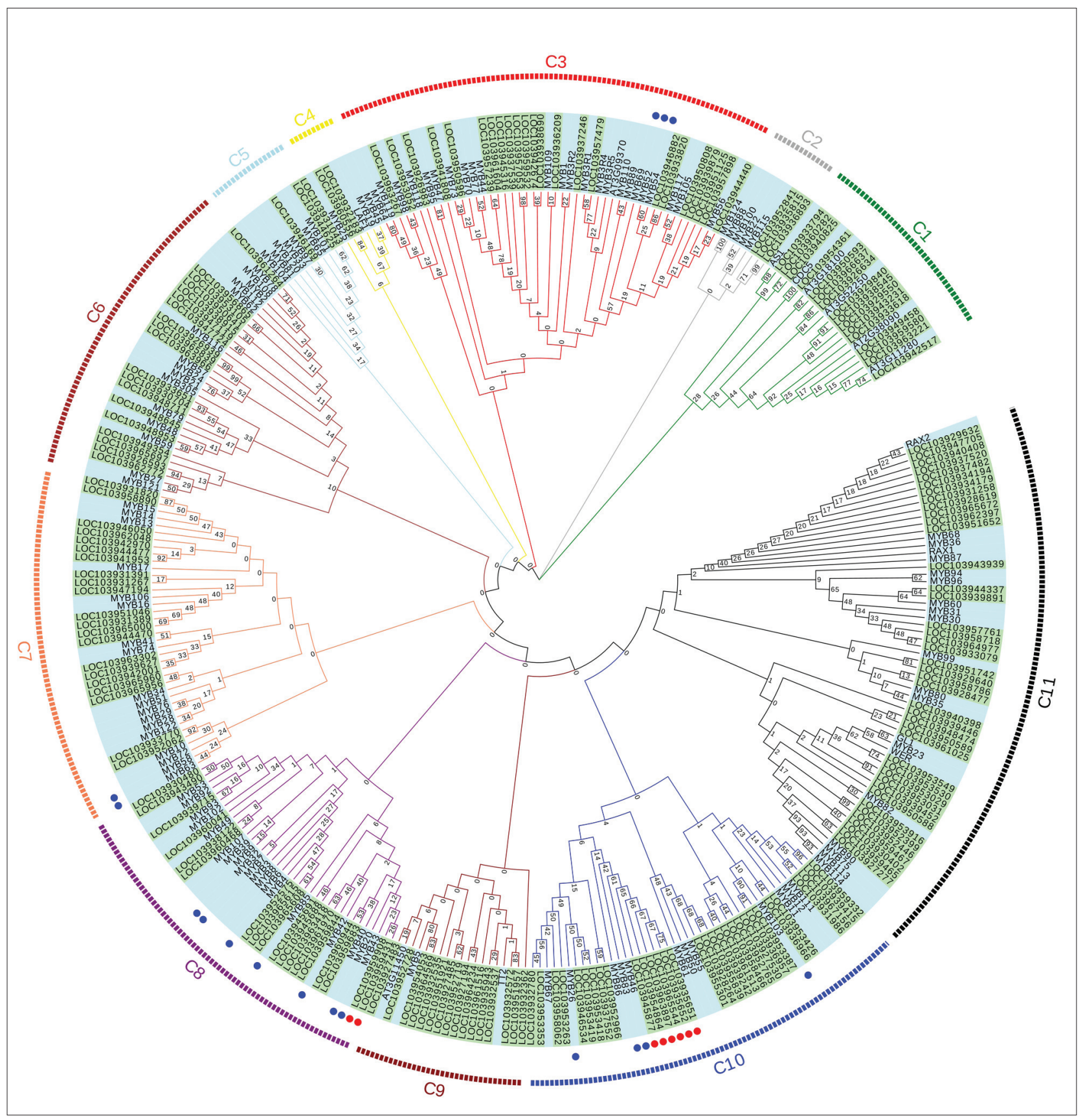

FIGURE 2. Phylogenetic relationships of the MYB family genes in P. bretschneideri and A. thaliana. Full-length amino acid sequences were aligned with MUSCLE program, the phylogenetic tree was constructed using MEGA-X software package. The phylogenetic trees were derived using the neighbor-joining (NJ) method with bootstrap value of 1,000 replicates. The tree divided the PbMYBs into 11 subgroups represented by different colored clades (C1-C11) within the tree. The MYB family genes annotated as associated with SCW biogenesis in pear labeled with blue circles, in $A$. thaliana labeled with red circles on the gene IDs in the figure. 


\section{Expression pattern analysis}

The fruit samples were collected from 20-year-old Korla pear trees (P. sinkiangensis Yü) grown in an orchard in Korla (Xinjiang, China) at three developmental stages (20, 50 and 80 days after flowering, ADF). They are representing prime (named as Day20 samples), late (Day50) and stationary stage (Day80) of stone cell differentiation in pear fruits, respectively (Mamat et al., 2020). Each sampling stage contains three biological replicates. Each biological replicate was the mixed sample of ten fruits collected from three pear trees. The fruit skins were peeled and pulps were sampled, then flash frozen in liquid nitrogen, ground into powder, and stored at $-80^{\circ} \mathrm{C}$ prior to transcriptome sequencing (Novogene Bioinformatics Technology Co. Ltd., Beijing, China).

The sequenced data (raw reads) was in fastq format. After quality control, the clean reads were aligned back to the pear reference genome with Hisat2 v.2.0.5. The read counts of genes in each sample were estimated, the expression abundance of genes were normalized and calculated by uniquely mapped reads with FPKM (fragments per kilobase of exon per million fragments mapped) method. Heatmap.2 function in Gplots package was used to analyze the gene expression pattern in fruit samples from different developmental stages.

\section{Quantification of selected MYBs in rough-skinned fruits}

The rough-skin is phenotypically invisible in the early stage of fruit development, and appears gradually in the later stage of fruit development from late July to mid-August (Figure 1). Hence, the skin and pulp of normal and rough-skinned fruits at 120 DAF (mature stage) were collected. Each group contains three biological replicates. Each biological replicate was the mixed sample of ten fruits collected from three pear trees. The fruit skin and pulp were sampled separately. Fruit skin were evenly peeled with a peeler, while cylindrical samples were obtained from the pulp $15 \mathrm{~mm}$ in diameter, then frozen in then liquid nitrogen, ground into mixed powder and stored at $-80^{\circ} \mathrm{C}$ until further usage.

14 MYBs, that were homologous to SCW associated MYBs in Arabidopsis, were selected for PCR quantification of their expressions in rough-skinned fruits from Korla pear. The primers were designed with Oligo 7.0 software (Supplemental Table S1). The specificity of primers was tested by running the amplification products on agarose gel. $1 \mu \mathrm{g}$ of total RNA was used to synthesize the cDNA by using reverse transcriptase RR037A (Takara, U.S.A.). The Real-Time PCR was carried out in $20 \mu \mathrm{L}$ system that contains $1 \mu \mathrm{L}$ of forward and reverse primers of each, SYBR Green Real-Time PCR Master Mixes (Invitrogen, U.S.A.) and $1 \mu \mathrm{L}$ of cDNA templates on STEP ONE PLUS $^{\mathrm{TM}}$ Real-Time PCR System (Applied Biosystems, U.S.A.). The PCR cycling conditions were: $95^{\circ} \mathrm{C}$ for $15 \mathrm{~min}$ followed by 40 cycles of $95^{\circ} \mathrm{C}$ for $10 \mathrm{~s}, 58^{\circ} \mathrm{C}$ for $20 \mathrm{~s}$, and $72^{\circ} \mathrm{C}$ for $20 \mathrm{~s}$. Each sample had three replicates and the expression level of each target gene was normalized to internal reference gene of Tubulin. Relative expression levels were calculated by the method of $2^{-\Delta \Delta \mathrm{Ct}}$ (Livak and Schmittgen, 2001).

\section{Results}

\section{MYB family genes in pear and Arabidopsis}

All protein sequences encoded from pear and Arabidopsis genome were searched against MYB family domain HMM model, and found that 424 transcripts (encoded by 333 genes) and 435 transcripts (encoded by 243 genes) contain MYB protein domain, respectively. Based on $E$-value, the transcripts that were aligned to the domain model with the lowest value among those transcripts that transcribed by the same gene, was chosen as a representative MYB encoded by that particular gene. The selected transcripts were also searched against SAMRT and Pfam database for further confirming the presence of the domain in these sequences. Furthermore, the confirmed transcripts were aligned to CDD database and found 193 transcripts in pear and 142 transcripts in Arabidopsis that contain MYB complete domain structure (Supplemental Tables S2 and S3).

\section{MYB family genes related to SCW biogenesis in pear fruit}

By annotating $P b M Y B$ genes against GO database at level eight, eight genes (namely LOC103945877, LOC103935652, LOC103963897, LOC103959908, LOC103959431, LOC103954864, LOC103965544 and LOC103935651) were enriched into the biological process of SCW biogenesis. In Arabidopsis, three master switches (MYB26, MYB46 and $M Y B 83$ ) and 13 downstream TFs (MYB4, MYB7, MYB20, MYB32, MYB42, MYB43, MYB52, MYB54, MYB58, MYB63, $M Y B 69$, MYB85 and MYB103) were reported to be associated with this process (Mitsuda et al., 2005; Yang et al., 2007; McCarthy et al., 2009; Zhou et al., 2009; Hussey et al., 2013; Scully et al., 2016; Guo et al., 2017). By multiple sequence alignment and phylogenetic tree construction, the homologous relation between the sequences of MYB genes of pear and Arabidopsis was primarily established (Figure 2). The SCW associated MYBs in Arabidopsis and their homolog genes in pear were clustered into the clade (C) of C3, C7, C8 and C10. The homolog sequences of SCW associated AtMYBs in pear genome were listed in Table 1. Among them, five genes were homologous to Arabidopsis SCW regulatory master switch MYB26, and six of them were homologous to $M Y B 83$, which has high sequence similarity to master switch MYB46. Besides, 22 PbMYBs were homologues to Arabidopsis SCW biogenesis regulatory network downstream TFs, includes AtMYB4 (three PbMYBs homologous to AtMYB4), AtMYB22 (two PbMYBs), AtMYB42 (one PbMYB), AtMYB43 (two PbMYBs), AtMYB52 (four PbMYBs), AtMYB58 (one PbMYB), AtMYB63 (one PbMYBs), AtMYB85 (four PbMYBs) and $A t M Y B 103$ (four $P b M Y B s$ ), respectively.

\section{Gene structure and motif distribution of SCW biogenesis related MYB homologs}

All sequences assigned to the clade C3, C7, C8 and C10 from the phylogenetic tree (Figure 2) were extracted, as they are including all the SCW related genes in pear and Arabidopsis. And their genetic relation, gene structure and sequence motif distribution were further visualized (Figure 3). By multiple sequence alignment and reconstructing the phylogenetic relation, these genes were divided into eight subgroups. In subgroup I, the pear gene LOC103959431, LOC103959908, LOC103945688 and LOC103945691 were closely located to the gene MYB43 and MYB20 of Arabidopsis. The blast result showed that the gene LOC103959431 and LOC103959908 have the highest sequence similarity to MYB20 (Table 1). Pear gene LOC103963418, LOC103959844 and LOC103961970 were clustered together with MYB42. Meanwhile, gene LOC103952062 and LOC103933770 were clustered with and blasted with the highest identity to MYB58 and MYB63 of Arabidopsis, respectively. In subgroup III, the pear gene LOC103965608 and LOC103965615 clustered with the SCW associated gene MYB4, MYB7 and MYB32 in Arabidopsis. The blast result indicated that they were highly identical to the sequence of MYB4 of Arabidopsis. In 
subgroup V, pear gene LOC103936957 and LOC103953387 clustered together and have the highest identity to MYB103 in Arabidopsis. All three SCW master switches of Arabidopsis and their homologs in pear were clustered into subgroup VI, gene LOC103935652, LOC103963897, LOC103935651 and LOC103965544 clustered with master switch MYB46, and gene LOC103954864 and LOC103945877 clustered with and have the highest identity to AtMYB83. Pear gene LOC103932780, LOC103953418, LOC103953419 and LOC103946534 were clustered with the master switch AtMYB26, among them, last three genes blasted with the highest similarity. Last but not least, the pear gene LOC103933820, LOC103936882, LOC103957898 and LOC103951125 were clustered together with Arabidopsis SCW biogenesis network downstream regulator MYB52, MYB54 and MYB69 in subgroup VIII. It is interesting to note that all those aforementioned homologs tend to have similar gene structures and motifs distributions except for LOC103932780, which has the rather low sequence identity to MYB26, with the short of motif 4 in its amino acid sequence compared with the neighbor sequences in the phylogenetic tree.

\section{The expression clustering of $M Y B s$ in pear fruits}

After the quality control of sequenced data, the clean reads were mapped back to the reference genome for gene expression analysis. The result showed that, among the 193 MYB members identified in pear genome in this study, 142 of them had expression at different developmental stages of Korla pear fruits. Genes with similar patterns of mRNA expression frequently have similar functions (Eisen et al., 1998; Brown et al., 2000; Wu et al., 2002). Thus, we have used Heatmap.2 function of Gplots package, clustered the expression value (FPKM) of these genes. When the dendrogram was cut with the height of 1.5 (Figure 4A), the gene expressions clustered into 10 different patterns among these genes during the fruit development (Figure 4B). In cluster I, thirteen genes showed relatively higher expressions in the samples from group Day20 and Day50 compared with group Day80. In cluster III, there were 21 genes that had higher expression in the fruits from 50 DAF in contrast with the samples of group Day20 and Day80. In cluster V, ten genes were highly expressed in Day80. In cluster VII, 43 MYB members were clustered together as they were highly expressed in Day20

TABLE 1. Homologous sequences of SCW related AtMYBs in pear.

\begin{tabular}{|c|c|c|c|c|c|c|}
\hline \multirow{2}{*}{ No. } & \multirow{2}{*}{ P. bretschneideri gene ID } & \multicolumn{2}{|c|}{ A. thaliana } & \multirow{2}{*}{ Identity } & \multirow{2}{*}{ E-value } & \multirow{2}{*}{ Score } \\
\hline & & Gene name & Gene ID & & & \\
\hline 1 & LOC103953418 & MYB26 & AT3G13890 & $88.19 \%$ & 1.59E-77 & 236 \\
\hline 2 & LOC103953419 & MYB26 & AT3G13890 & $88.19 \%$ & 1.59E-77 & 236 \\
\hline 3 & LOC103946534 & MYB26 & AT3G13890 & $88.19 \%$ & $1.71 \mathrm{E}-77$ & 236 \\
\hline 4 & LOC103953353 & MYB26 & AT3G13890 & $75.59 \%$ & $9.86 \mathrm{E}-67$ & 207 \\
\hline 5 & LOC103958062 & MYB26 & AT3G13890 & $56.06 \%$ & $1.04 \mathrm{E}-66$ & 207 \\
\hline 6 & LOC103954864 & MYB83 & AT3G08500 & $47.49 \%$ & $3.66 \mathrm{E}-83$ & 249 \\
\hline 7 & LOC103945877 & MYB83 & AT3G08500 & $46.07 \%$ & $1.53 E-82$ & 247 \\
\hline 8 & LOC103935651 & MYB83 & AT3G08500 & $43.57 \%$ & $1.57 E-76$ & 232 \\
\hline 9 & LOC103935652 & MYB83 & AT3G08500 & $43.57 \%$ & $1.57 \mathrm{E}-76$ & 232 \\
\hline 10 & LOC103963897 & MYB83 & AT3G08500 & $43.31 \%$ & $1.87 \mathrm{E}-75$ & 229 \\
\hline 11 & LOC103965544 & MYB83 & АТЗG08500 & $42.33 \%$ & $2.75 E-74$ & 226 \\
\hline 12 & LOC103965608 & MYB4 & AT4G38620 & $62.63 \%$ & $2.4 \mathrm{E}-117$ & 330 \\
\hline 13 & LOC103965615 & MYB4 & AT4G38620 & $62.63 \%$ & $2.4 \mathrm{E}-117$ & 330 \\
\hline 14 & LOC103952575 & MYB4 & AT4G38620 & $61.06 \%$ & $2.31 \mathrm{E}-91$ & 266 \\
\hline 15 & LOC103959908 & MYB20 & AT1G66230 & $61.91 \%$ & 2.3E-101 & 291 \\
\hline 16 & LOC103959431 & MYB20 & AT1G66230 & $58.55 \%$ & 4.23E-97 & 280 \\
\hline 17 & LOC103963418 & MYB42 & AT4G12350 & $53.07 \%$ & 7.49E-89 & 258 \\
\hline 18 & LOC103960944 & MYB43 & AT5G16600 & $61.41 \%$ & $1.55 \mathrm{E}-81$ & 242 \\
\hline 19 & LOC103959683 & MYB43 & AT5G16600 & $60.87 \%$ & 7.74E-81 & 240 \\
\hline 20 & LOC103946882 & MYB52 & AT1G17950 & $55.36 \%$ & $2.68 \mathrm{E}-90$ & 261 \\
\hline 21 & LOC103933820 & MYB52 & AT1G17950 & $52.02 \%$ & 4.38E-81 & 238 \\
\hline 22 & LOC103957898 & MYB52 & AT1G17950 & $83.64 \%$ & $1.01 \mathrm{E}-67$ & 207 \\
\hline 23 & LOC103951125 & MYB52 & AT1G17950 & $82.73 \%$ & $2.31 \mathrm{E}-67$ & 206 \\
\hline 24 & LOC103952062 & MYB58 & AT1G16490 & $44.88 \%$ & 1.34E-78 & 236 \\
\hline 25 & LOC103933770 & MYB63 & AT1G79180 & $57.52 \%$ & 1.33E-59 & 189 \\
\hline 26 & LOC103945688 & MYB85 & AT4G22680 & $55.99 \%$ & 2.6E-102 & 293 \\
\hline 27 & LOC103945691 & MYB85 & AT4G22680 & $55.99 \%$ & 2.6E-102 & 293 \\
\hline 28 & LOC103959844 & MYB85 & AT4G22680 & $51.70 \%$ & $1.26 \mathrm{E}-89$ & 260 \\
\hline 29 & LOC103961970 & MYB85 & AT4G22680 & $52.20 \%$ & 1.43E-89 & 260 \\
\hline 30 & LOC103936957 & MYB103 & AT1G63910 & $51.77 \%$ & 2.6E-106 & 309 \\
\hline 31 & LOC103953387 & MYB103 & AT1G63910 & $50.81 \%$ & $4 \mathrm{E}-104$ & 304 \\
\hline 32 & LOC103953426 & MYB103 & AT1G63910 & $84.03 \%$ & $5.74 \mathrm{E}-76$ & 231 \\
\hline 33 & LOC103936966 & MYB103 & AT1G63910 & $83.19 \%$ & 7.61E-73 & 229 \\
\hline
\end{tabular}


compared with samples from two later stages in question. In cluster VIII, eighteen genes also showed higher expression in Day20, but some of them have little or no expression in some replicated of group Day20. The rest of the MYB members clustered to cluster II, IV, VI, IX and X showed no constant expression in all biological replicates of fruit samples at any developmental stages.

Identification of SCW biogenesis related PbMYBs in pear

We have selected MYB family members from the expression pattern cluster I, VII and VIII, as they were highly expressed in pear fruits $20 \mathrm{DAF}$ and/or $50 \mathrm{DAF}$ compared with the samples that were collected at stone cell differentiation stationary period. The differential expression of these genes was analyzed (Table 2). The results showed that six of them were significantly upregulated $\left(P_{\text {adj }}\right.$-value $\left.<0.05\right)$ in group Day20 and Day50 samples in cluster I. In cluster VII, 26 pear MYBs were differentially upregulated $\left(P_{\text {adj }}\right.$-value $\left.<0.05\right)$ in Day20 samples and most of them also showed upregulation $\left(P_{\text {adj }}\right.$-value $\left.<0.05\right)$ in Day50 samples. It is interesting to note that 13 of these genes were highly homologous to those of SCW master switches, such as $M Y B 83$, and regulatory network downstream transcription factor MYB20, MYB42, MYB52, MYB85 and MYB103 in Arabidopsis. In cluster VIII,

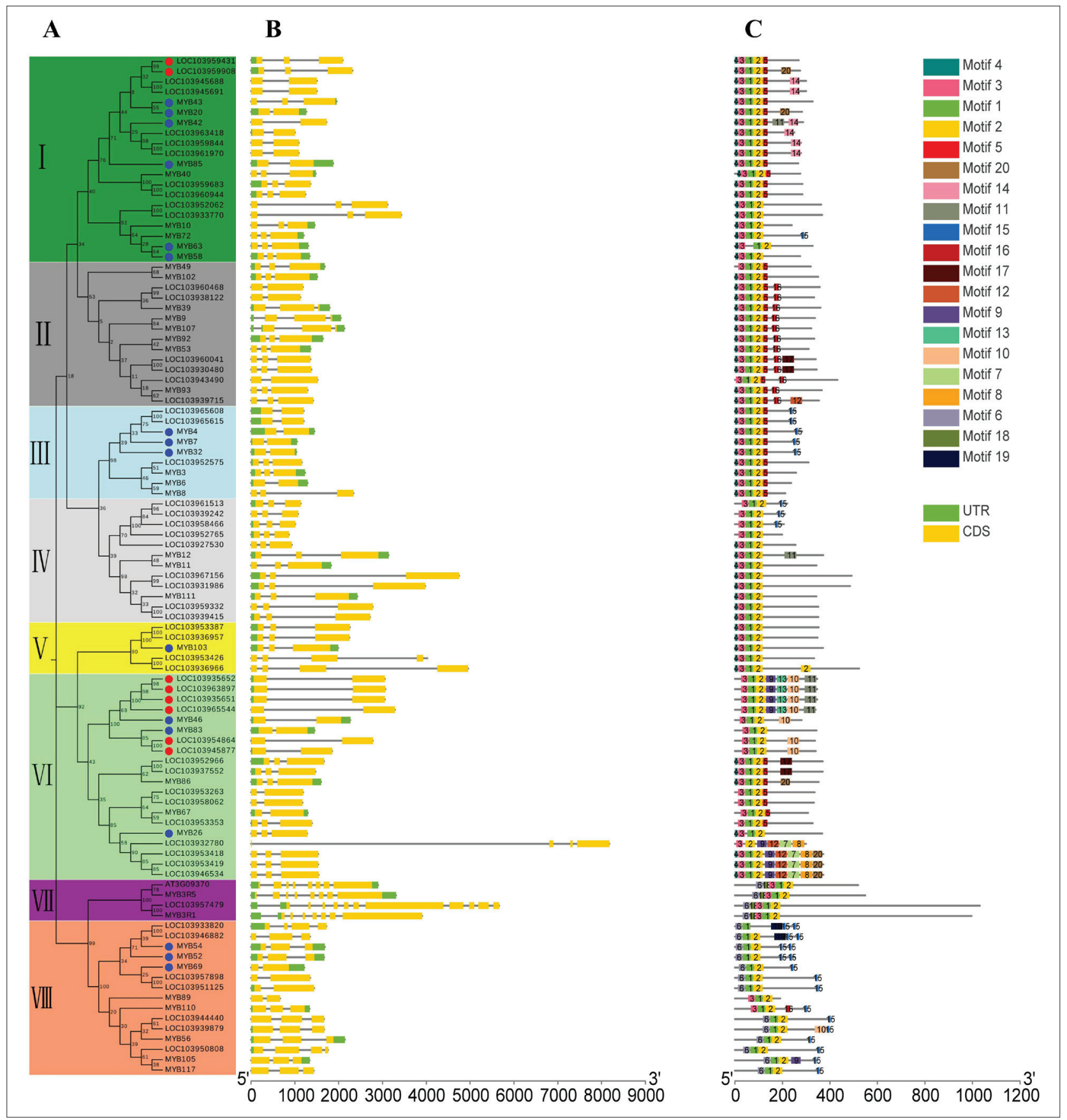

FIGURE 3. Phylogenetic relationships, gene structures, and architecture of conserved protein motifs in $99 M Y B$ genes in P. bretschneideri and A. thaliana. A is the phylogenetic tree that constructed by MEGA-X using the NJ method. Eight subgroups on the phylogenetic tree were shown by different background colors. B is the structures of MYB genes, green boxes indicate UTR regions, yellow boxes indicate exons and black lines indicate introns. C is the motifs distribution of MYB proteins. Different motifs were indicated by different colors for motif 1 to 20 . The sequence information for each motif is provided in Supplemental Table S4. 
pear gene LOC103945688 was upregulated $\left(P_{\text {adj }}\right.$-value $\left.<0.05\right)$ in the fruits from Day20 and Day50 samples, which is a homolog of MYB85 from Arabidopsis.

\section{Quantification of SCW related MYBs in the rough- skinned fruits}

The fourteen homologs of SCW associated AtMYBs in pear, which were differentially upregulated at the key stages of stone cell differentiation in pear fruits, were chosen for the quantification of their expression in rough-skinned fruits from ripening stage. During the experiment, the expression levels of gene LOC103936957, LOC103953387,
LOC103945877 and LOC103963897 could not be quantified, this may be because they had a very low expression or not expressed at the mature stage in pear fruits. Among the rest of $10 \mathrm{PbMYBs}$, seven genes were highly expressed in either or both the peel and pulp of rough-skinned fruits (Figure 5). Pear gene LOC103963418 and LOC103945688 were upregulated more than 2.58 folds in both the peel and pulp of rough-skinned fruits compared with normal fruits, and they are homologous to MYB42 and MYB85 of Arabidopsis, respectively. Gene LOC103959908 and LOC103959431 were upregulated in the peel of rough-skinned fruits around 2.26 folds compared with normal fruits, which are the homologs
A

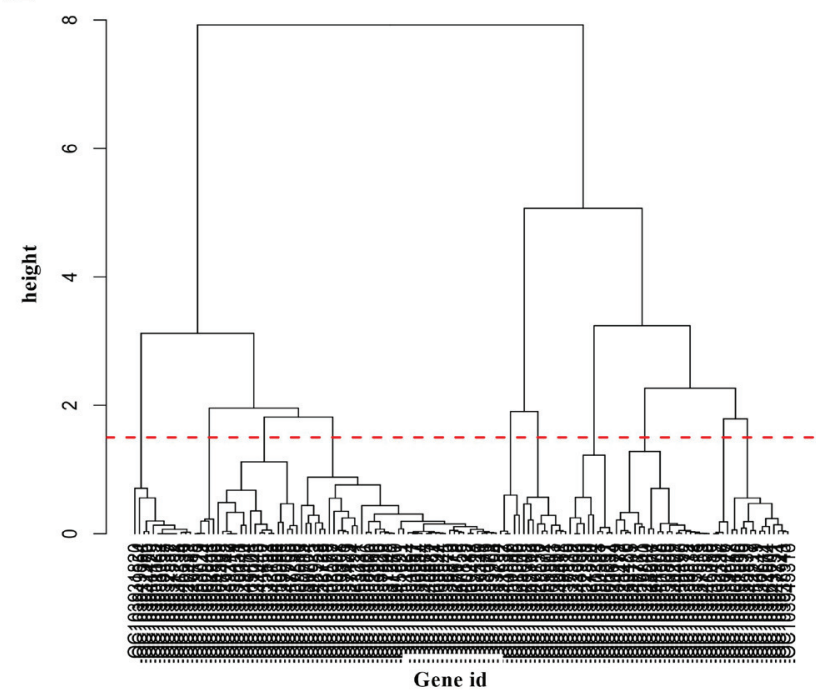

B

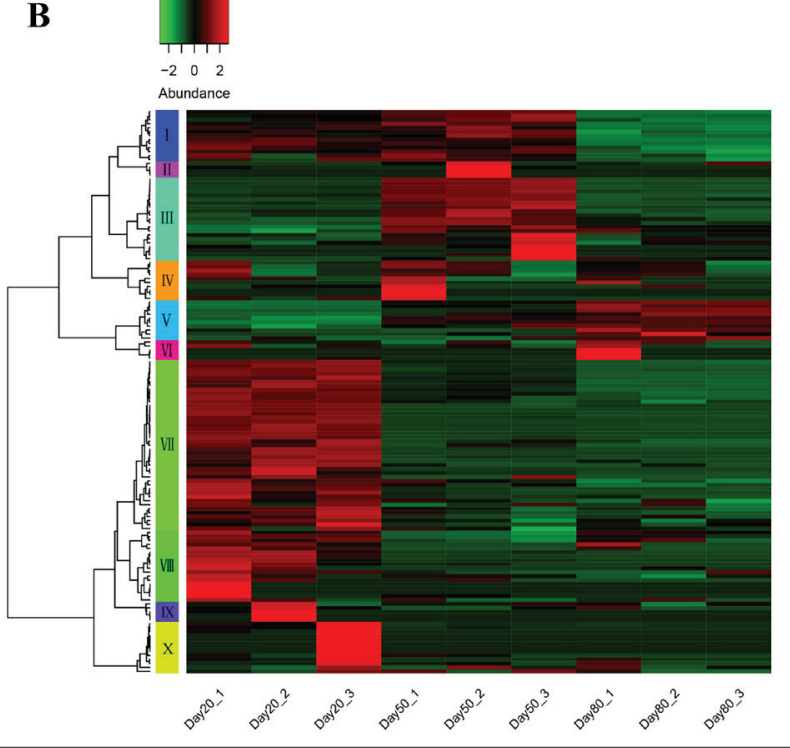

FIGURE 4. Expression clustering of MYB family genes in transcriptomes of Korla pear fruits at different developmental stages. $A$ is the dendrogram of FPKM value clusters of MYB family genes; the vertical axis represents the height of the dendrogram, the horizontal axis represents the genes in different clusters. B is the gene expression heat map; vertical axis represents 10 branches after cutting the dendrogram, the horizontal axis represents different samples that collected from different fruit developmental stages; red and green colors in the heat map represent the high and low levels of expression of $P b M Y B s$, respectively.

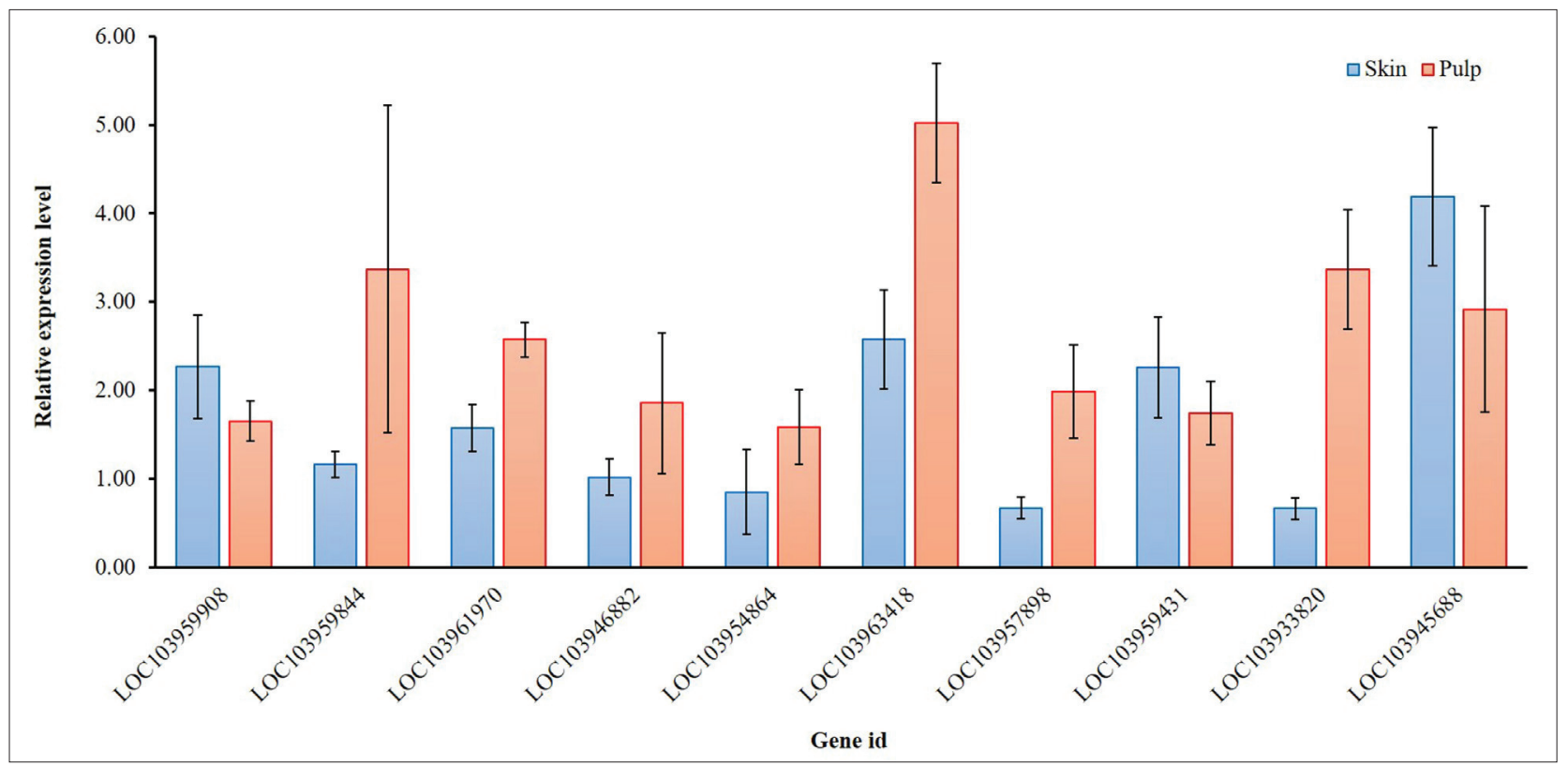

FIGURE 5. Quantification of SCW-related PbMYBs expression in rough-skinned fruits. Blue column represents the gene expression level in the fruit skin and pink column represents the gene expression level in the fruit pulp. The bar height represents the mean expression values of three replicates \pm standard deviation (SD). 
TABLE 2. Potential SCW biogenesis related MYB family genes in pear.

\begin{tabular}{|c|c|c|c|c|c|}
\hline \multirow{2}{*}{ Gene ID } & \multicolumn{2}{|c|}{ Day20 vs. Day80 } & \multicolumn{2}{|c|}{ Day50 vs. Day80 } & \multirow{2}{*}{ Cluster } \\
\hline & LogFC & $P_{\text {adj }}$-value & LogFC & $P_{\text {adj }}$-value & \\
\hline LOC103952719 & 5.71 & $1.1 \mathrm{E}-78$ & 5.40 & $1.6 \mathrm{E}-85$ & I \\
\hline LOC103942517 & 2.23 & 9.3E-07 & 1.90 & $3.9 \mathrm{E}-04$ & I \\
\hline LOC103945316 & 0.91 & $6.8 \mathrm{E}-06$ & 1.27 & $1.9 \mathrm{E}-11$ & I \\
\hline LOC103950641 & 1.50 & $3.4 \mathrm{E}-04$ & 1.83 & 4.5E-05 & I \\
\hline LOC103935943 & 1.62 & $8.5 \mathrm{E}-04$ & 2.43 & $1.6 \mathrm{E}-09$ & I \\
\hline LOC103947724 & 0.91 & 2.7E-02 & 1.52 & $2.2 \mathrm{E}-06$ & I \\
\hline LOC103936957• & 29.78 & $1.6 \mathrm{E}-86$ & 24.02 & 3.3E-02 & VII \\
\hline LOC103953387• & 29.48 & $7.2 \mathrm{E}-75$ & 24.13 & $5.4 \mathrm{E}-02$ & VII \\
\hline LOC103963897• & 29.01 & $3.1 \mathrm{E}-63$ & 24.80 & 5.7E-04 & VII \\
\hline LOC103959908• & 4.97 & $3.6 \mathrm{E}-54$ & 2.75 & 4.0E-19 & VII \\
\hline LOC103959844• & 7.42 & $1.8 \mathrm{E}-51$ & 3.47 & 5.6E-06 & VII \\
\hline LOC103961970• & 6.90 & 5.7E-33 & 3.19 & 2.6E-05 & VII \\
\hline LOC103946882• & 8.83 & $1.2 \mathrm{E}-31$ & 4.85 & 7.4E-02 & VII \\
\hline LOC103954864• & 7.84 & $1.4 \mathrm{E}-30$ & 3.23 & 4.1E-01 & VII \\
\hline LOC103940825 & 2.91 & $1.2 \mathrm{E}-26$ & 1.61 & $1.0 \mathrm{E}-05$ & VII \\
\hline LOC103945877• & 6.38 & $2.3 \mathrm{E}-25$ & 1.00 & $9.5 \mathrm{E}-01$ & VII \\
\hline LOC103963418• & 4.29 & $5.3 \mathrm{E}-25$ & 2.86 & $2.1 \mathrm{E}-16$ & VII \\
\hline LOC103957479 & 2.98 & 7.7E-23 & 0.63 & 3.7E-01 & VII \\
\hline LOC103937520 & 5.44 & $8.5 \mathrm{E}-22$ & 3.62 & 5.1E-06 & VII \\
\hline LOC103933079 & 2.01 & $9.4 \mathrm{E}-22$ & 0.58 & 7.2E-02 & VII \\
\hline LOC103957898• & 6.13 & 3.2E-20 & 2.50 & $1.8 \mathrm{E}-01$ & VII \\
\hline LOC103933794 & 1.58 & $1.5 \mathrm{E}-15$ & 0.63 & 1.6E-02 & VII \\
\hline LOC103960282 & 3.30 & $5.4 \mathrm{E}-15$ & 1.82 & $6.4 \mathrm{E}-04$ & VII \\
\hline LOC103967156 & 3.65 & $1.8 \mathrm{E}-14$ & 2.19 & 4.5E-03 & VII \\
\hline LOC103952966 & 7.51 & $3.8 \mathrm{E}-13$ & 2.88 & 8.3E-02 & VII \\
\hline LOC103959431• & 6.48 & $5.4 \mathrm{E}-12$ & 4.49 & $1.8 \mathrm{E}-02$ & VII \\
\hline LOC103928619 & 4.19 & $6.3 \mathrm{E}-11$ & 2.45 & 1.4E-01 & VII \\
\hline LOC103938545 & 1.22 & 2.2E-09 & -0.14 & 5.1E-01 & VII \\
\hline LOC103927194 & 3.61 & $2.2 \mathrm{E}-07$ & 2.51 & 2.5E-02 & VII \\
\hline LOC103958466 & 5.43 & 2.4E-06 & 2.93 & 2.2E-01 & VII \\
\hline LOC103950808 & 1.67 & 4.6E-04 & 0.19 & $1.0 \mathrm{E}+00$ & VII \\
\hline LOC103933820• & 3.67 & $1.3 \mathrm{E}-03$ & -2.59 & 8.3E-01 & VII \\
\hline LOC103945688• & 27.86 & 1.7E-12 & 24.27 & $6.3 \mathrm{E}-02$ & VII \\
\hline
\end{tabular}

- indicates the PbMYBs that are highly homologous with SCW associated MYBs in Arabidopsis.

of AtMYB20. The gene LOC103959844, LOC103961970 and LOC103933820 were upregulated more than 2.57 folds in the pulp of rough-skinned fruits. Among them, the former two genes are the homologs of MYB85 and the last one is homologous to MYB52 of Arabidopsis.

\section{Discussion}

Currently, formation of rough-skinned fruits is one of the major factors that adversely affects the fruit quality in Korla pear (Pyrus sinkiangensis Yü). Increased stone cell content is one of the typical characteristics of rough-skinned fruits. Stone cells are formed by secondary thickening of parenchyma cell walls, which consist of a large amount of lignin, cellulose and hemicellulose (Nie et al., 2009; Brahem et al., 2017; Mamat et al., 2019). Being a type of sclerenchyma, as fibers, stone cell differentiation must be carried out by cell autonomous processes (Smith et al., 2013). Being the most prevalent TFs in plants, the MYBs regulate the coordinated activation of secondary wall biosynthetic genes (Mitsuda et al., 2005; Zhong et al., 2010; Yang and Wang, 2016). In this work, we identified 14 DEGs encoding different MYB mem- bers in pear that are highly homologous to a battery of SCW associated MYBs in Arabidopsis, including MYB46/83, MYB20, MYB42, MYB52, MYB85 and MYB103, all of which specifically expressed in secondary wall-forming cells (Zhong et al., 2008; McCarthy et al., 2009). Among these DEGs, the gene LOC103954864, LOC103945877 and LOC103963897 were homologs of AtMYB46/AtMYB83, as the second-level master switches, regulates a series of downstream $M Y B s$ and some direct target genes that involve in the biosynthesis of secondary cell walls (Zhong et al., 2007; McCarthy et al., 2009). All of these three genes were abundantly expressed at the prime stage of stone cell differentiation, with higher fold changes in expression between prime and stationary stages of stone cell differentiation, indicating that these two TFs may play critical roles in the process of secondary thickening of cell walls in pear stone cells. Supporting this, both AtMYB46 and AtMYB83 genes and their close homologs in other plants have been reported to express in secondary wall-forming cells, induce ectopic secondary wall deposition when overexpressed, and mutation or repression of these genes causes a defective secondary wall phenotype (Zhong et al., 2008; 
McCarthy et al., 2009; Zhou et al., 2009; Zhong et al., 2011; Zhong and Ye, 2015). In addition, secondary wall-associated cellulose synthases genes (CESA4, CESA7 and CESA8), xylan biosynthesis genes (IRX8,IRX9, IRX14, and IRX15-L) and some lignin synthetic genes have also been reported to be upregulated by MYB46 through directly binding the promoter region of these genes (Kim et al., 2012, 2013, 2014; Zhong and Ye, 2012). All these genes showed upregulation during the prime stage of stone cell differentiation, and the stone cell components (cellulose, hemicellulose and lignin) also peaked during this period in pear fruits (Mamat et al., 2020), suggesting that pear homologs of AtMYB46 and AtMYB83 might play important regulatory functions during stone cell differentiation in Korla pear fruits.

After being induced, AtMYB83 and AtMYB46 trigger the expression of downstream MYBs (Zhong et al., 2007; McCarthy et al., 2009). In this work, we found 11 DEGs that are homologous to MYB20, MYB42, MYB52, MYB85 and MYB103 in A. thaliana. All of these genes showed peak expression at the prime stage of stone cell differentiation, then decreased rapidly in later stages. It was consistent with the tendency of changes in stone cells and lignin in Korla pear fruits (Mamat et al., 2019, 2020). MYB85 is recognized as lignin-specific transcriptional activator, which positively regulates various lignin synthesis genes (Zhong et al., 2007, 2008; McCarthy et al., 2009; Zhou et al., 2009). Dominant repression or overexpression of MYB85 results in a specific repression or activation of lignin biosynthesis and concomitant ectopic deposition in both vessel and fiber cells (Zhong et al., 2008; Zhou et al., 2009). It is reported that MYB20 and MYB42 regulate lignin biosynthesis by direct activation of phenylalanine and lignin synthetic genes. Similar to MYB85, disruption of these genes caused lignin defect phenotype in Arabidopsis (Zhong et al., 2008; Zhou et al., 2009; Geng et al., 2020). Accordingly, synchronizing with the lignin accumulation period (Mamat et al., 2019), all homologs of AtMYB85, AtMYB20 and AtMYB42 in pear showed peak expression in the prime stage of stone cell differentiation, and reached the lowest level in the stationary stage in pear fruits, suggesting that the homologs of MYB20 and MYB42 together with $M Y B 85$ may play crucial regulatory functions during lignin accumulation in Korla pear fruits.

MYB52 and MYB103 are also reported to be the secondary wall-associated $M Y B s$. In our transcriptome data, three homologs of the AtMYB103 were differentially expressed during the different stages of pear fruit development, with the highest expression shown in prime stage of stone cell differentiation. In Arabidopsis, the overexpression of MYB103 results in increased, but otherwise (repression or mutation) loss of SCW in fibers (Zhong et al., 2008). Similar phenotype was observed in plants with dominant repression of MYB52, but the overexpression of MYB52 was unable to induce the ectopic secondary wall deposition, just slightly induce CESA8, IRX9 and 4CL1 (Zhong et al., 2008; Hao and Mohnen, 2014). It is also reported that MYB103 regulates the expression of CesA8, indicating that transcription factor MYB103 may affect the SCW thickening of cell walls by the regulation of cellulose biosynthesis.

Stone cells are commonly present in all pear varieties, and appear at cell division period during normal fruit development. At mature stage, they are nearly invisible in fruits of all cultivated pear varieties under normal conditions (Nie et al, 2009; Jin et al., 2013; Yan et al., 2014; Mamat et al., 2019). As for the rough-skinned fruits, also known as hard-end fruits in 'Whangkeumbae' pear (Pyrus pyrifolia), it is a physi- ological disorder that has become increasingly common in recent years, resulting in considerable economic loss to the pear growers. And the increased stone cell content is a main cause of hard-end disorder in pear fruits (Lu et al., 2014; Li et al., 2017, 2019). All the SCW associated MYBs that differentially expressed in this study were abundantly expressed at the prime stage of stone cell differentiation, then expression levels started to decrease gradually. This trend is similar with the changing trends of cellulose, hemicellulose and lignin content during the fruit development. Furtherly, the homologs of AtMYB20, AtMYB42, AtMYB52 and AtMYB85 were upregulated in either or both the skin and pulp of roughskinned fruits. These MYBs are the downstream TFs that regulate the biosynthesis of SCW components in Arabidopsis. Hence, the upregulation of these PbMYBs in rough-skinned fruits indicate that they may play crucial roles in the process of stone cell differentiation or fruit skin roughening on Korla pear fruits.

\section{Conclusions}

In this work, 193 MYB family genes were identified in pear genome. Among them, thirty-three were closely homologous to SCW associated MYBs in Arabidopsis. During the stone cell differentiation in pear fruits, fourteen $P b M Y B S$ which are homologs of SCW related AtMYBs (namely MYB20, MYB42, MYB52, MYB83, MYB85 and MYB103), showed peak expression in the prime stage of stone cell differentiation, and down regulated gradually in later stages. Furthermore, pear homologs of AtMYB20, AtMYB42, AtMYB52 and AtMYB85 were significantly upregulated in either or both of the skin and pulp of rough-skinned fruits from ripening stage. These results indicated that these SCW associated PbMYBs play fundamental roles during the stone cell differentiation and rough-skin formation in pear fruits.

\section{Acknowledgments}

This research was supported by National Natural Science Foundation of China (Grant No. 31760565) and Basic Scientific Research Supporting Program of the Autonomous Region Public Welfare Research Institutes (Grant No. KY2020113). The funders had no role in study design, data collection and analysis, decision to publish, or preparation of the manuscript.

\section{Data availability statement}

The transcriptome sequencing (RNA-seq) data supporting the results of this work can be accessed in the Short Read Archive (SRA) of National Center for Biotechnology Information (NCBI) with the accession number SRX7122321 - SRX7122329.

\section{References}

Baumann, K., Perez-Rodriguez, M., Bradley, D., Venail, J., Bailey, P., Jin, H., Koes, R., Roberts, K., and Martin, C. (2007). Control of cell and petal morphogenesis by R2R3 MYB transcription factors. Development 134, 1691-1701. https://doi.org/10.1242/dev.02836.

Brahem, M., Renard, C.M., Gouble, B., Bureau, S., and Le Bourvellec, C. (2017). Characterization of tissue specific differences in cell wall polysaccharides of ripe and overripe pear fruit. Carbohydr. Polym. 156, 152-164. https://doi.org/10.1016/j.carbpol.2016.09.019.

Brown, M.P., Grundy, W.N., Lin, D., Cristianini, N., Sugnet, C.W., Furey, T.S., Ares, M. Jr., and Haussler, D. (2000). Knowledge-based analysis of microarray gene expression data by using support vector machines. Proc. Natl. Acad. Sci. U.S.A. 97, 262-267. https://doi.org/10.1073/ pnas.97.1.262. 
Cao, Y., Han, Y., Li, D., Lin, Y., and Cai, Y. (2016a). MYB transcription factors in chinese pear (Pyrus bretschneideri Rehd.): Genome-wide identification, classification, and expression profiling during fruit development. Front Plant Sci. 7, 577. https://doi.org/10.3389/ fpls.2016.00577.

Cao, Y., Han, Y., Li, D., Lin, Y., and Cai, Y. (2016b). Systematic analysis of the 4-coumarate: coenzyme A ligase (4CL) related genes and expression profiling during fruit development in the Chinese pear. Genes (Basel) 7, 89. https://doi.org/10.3390/genes7100089.

Chen, Y., Chao, Y., Tseng, T., Huang, C., Lo, P., and Lu, C. (2017). Two MYB-related transcription factors play opposite roles in sugar signaling in Arabidopsis. Plant Mol. Biol. 93, 299-311. https://doi. org/10.1007/s11103-016-0562-8.

Dubos, C., Stracke, R., Grotewold, E., Weisshaar, B., Martin, C., and Lepiniec, L. (2010). MYB transcription factors in Arabidopsis. Trends Plant Sci. 15, 573-581. https://doi.org/10.1016/j. tplants.2010.06.005.

Eisen, M.B., Spellman, P.T., Brown, P.O., and Botstein, D. (1998). Cluster analysis and display of genome-wide expression patterns. Proc. Natl. Acad. Sci. U.S.A. 95, 14863-14868. https://doi.org/10.1073/ pnas.95.25.14863.

Geng, P., Zhang, S., Liu, J., Zhao, C., Wu, J., Cao, Y., Fu, C., Han, X., He, H., and Zhao, Q. (2020). MYB20, MYB42, MYB43, and MYB85 regulate phenylalanine and lignin biosynthesis during secondary cell wall formation. Plant Physiol. 182, 1272-1283. https://doi.org/10.1104/ pp.19.01070.

Guo, H., Wang, Y., Wang, L., Hu, P., Wang, Y., Jia, Y., Zhang, C., Zhang, Y., Zhang, Y., Wang, C., and Yang, C. (2017). Expression of the MYB transcription factor gene BplMYB46 affects abiotic stress tolerance and secondary cell wall deposition in Betula platyphylla. Plant Biotechnol. J. 15, 107-121. https://doi.org/10.1111/pbi.12595.

Hajiebrahimi, A., Owji, H., and Hemmati, S. (2017). Genome-wide identification, functional prediction, and evolutionary analysis of the R2R3-MYB superfamily in Brassica napus. Genome 60, 797-814. https://doi.org/10.1139/gen-2017-0059.

Hao, Z., and Mohnen, D. (2014). A review of xylan and lignin biosynthesis: foundation for studying Arabidopsis irregular xylem mutants with pleiotropic phenotypes. Crit. Rev. Biochem. Mol. Biol. 49, 212-241. https://doi.org/10.3109/10409238.2014.889651.

Hou, X.J., Li, S.B., Liu, S.R., Hu, C.G., and Zhang, J.Z. (2014). Genomewide classification and evolutionary and expression analyses of citrus MYB transcription factor families in sweet orange. PLoS ONE 9, e112375. https://doi.org/10.1371/journal.pone.0112375.

Hussey, S.G., Mizrachi, E., Creux, N.M., and Myburg, A.A. (2013). Navigating the transcriptional roadmap regulating plant secondary cell wall deposition. Front. Plant Sci. 4, 325. https://doi.org/10.3389/ fpls.2013.00325.

Jin, Q., Yan, C., Qiu, J., Zhang, N., Lin, Y., and Cai, Y. (2013). Structural characterization and deposition of stone cell lignin in Dangshan $\mathrm{Su}$ pear. Sci. Hortic. 155, 123-130. https://doi.org/10.1016/j. scienta.2013.03.020.

Kim, W.C., Ko, J.H., and Han, K.H. (2012). Identification of a cis-acting regulatory motif recognized by MYB46, a master transcriptional regulator of secondary wall biosynthesis. Plant Mol. Biol. 78, 489501. https://doi.org/10.1007/s11103-012-9880-7.

Kim, W.C., Ko, J.H., Kim, J.Y., Kim, J., Bae, H.J., and Han, K.H. (2013). MYB46 directly regulates the gene expression of secondary wallassociated cellulose synthases in Arabidopsis. Plant J. 73, 26-36. https://doi.org/10.1111/j.1365-313x.2012.05124.x.

Kim, W.C., Kim, J.Y., Ko, J.H., Kang, H., and Han, K.H. (2014). Identification of direct targets of transcription factor MYB46 provides insights into the transcriptional regulation of secondary wall biosynthesis. Plant Mol. Biol. 85, 589-599. https://doi.org/10.1007/s11103-014-0205-x.
Ko, J.H., Kim, W.C., and Han, K.H. (2009). Ectopic expression of MYB46 identifies transcriptional regulatory genes involved in secondary wall biosynthesis in Arabidopsis. Plant J. 60, 649-665. https://doi. org/10.1111/j.1365-313X.2009.03989.x.

Li, M., Cheng, C., Zhang, X., Zhou, S., Wang, C., Ma, C., and Yang, S. (2019). PpNAC187 enhances lignin synthesis in 'Whangkeumbae' pear (Pyrus pyrifolia) 'hard-end' fruit. Molecules 24, 4338. https:// doi.org/10.3390/molecules24234338.

Li, W., Ding, Z., Ruan, M., Yu, X., Peng, M., and Liu, Y. (2017). Kiwifruit R2R3-MYB transcription factors and contribution of the novel AcMYB75 to red kiwifruit anthocyanin biosynthesis. Sci. Rep. 7, 16861. https://doi.org/10.1038/s41598-017-16905-1.

Li, X., Xue, C., Li, J., Qiao, X., Li, L., Yu, L., Huang, Y., and Wu, J. (2016). Genome-wide identification, evolution and functional divergence of MYB transcription factors in Chinese White Pear (Pyrus bretschneideri). Plant Cell Physiol. 57, 824-847. https://doi. org/10.1093/pcp/pcw029.

Li, Z., Yang, Z., and Wang, R. (2017). Relationship between 'hard end' and active oxygen metabolism in 'Whangkeumbae' pear fruit. Northern Hortic. 2017 (02), 1-6.

Lin-Wang, K., Bolitho, K., Grafton, K., Kortstee, A., Karunairetnam, S., McGhie, T.K., Espley, R.V., Hellens, R.P., and Allan, A.C. (2010). An R2R3 MYB transcription factor associated with regulation of the anthocyanin biosynthetic pathway in Rosaceae. BMC Plant Biol. 10, 50. https://doi.org/10.1186/1471-2229-10-50.

Liu, L., White, M.J., and MacRae, T.H. (1999). Transcription factors and their genes in higher plants functional domains, evolution and regulation. Eur. J. Biochem. 262, 247-257. https://doi.org/10.1046/ j.1432-1327.1999.00349.x

Livak, K.J., and Schmittgen, T.D. (2001). Analysis of relative gene expression data using real-time quantitative PCR and the $2^{-\triangle \Delta C T}$ method. Methods 25, 402-408. https://doi.org/10.1006/ meth.2001.1262.

Lu, G., Li, Z., Zhang, X., Wang, R., and Yang, S. (2014). Expression analysis of lignin-associated genes in Hard End pear (Pyrus pyrifolia Whangkeumbae) and its response to calcium chloride treatment conditions. J. Plant Growth Regul. 34, 251-262. https://doi. org/10.1007/s00344-014-9461-x.

Mamat, A., Ayup, M., Zhang, X., Ma, K., Mei, C., Yan, P., Han, L., and Wang, J. (2019). Pulp lignification in Korla fragrant pear. Eur. J. Hortic. Sci. 84, 263-273. https://doi.org/10.17660/eJHS.2019/84.5.2.

Mamat, A., Zhang, X., Ma, K., Mei, C., Yan, P., Han, L., and Wang, J. (2020). Study on the relationship between the stone cell formation and apoptosis during the fruit development of Korla Xiangli Pear. J. Fruit Sci. 37, 59-67. https://doi.org/10.13925/j.cnki.gsxb.20180494.

Mamat, A., Tusong, K., Xu, J., Yan, P., and Wang, J. (2021). Integrated transcriptomic and proteomic analysis reveals the complex molecular mechanisms underlying stone cell formation in Korla pear. Sci. Rep. (under revision)

Martin, C., and Paz-Ares, J. (1997). MYB transcription factors in plants. Trends Genet. 13, 67-73. https://doi.org/10.1016/s01689525(96)10049-4.

McCarthy, R.L., Zhong, R., and Ye, Z.H. (2009). MYB83 is a direct target of SND1 and acts redundantly with MYB46 in the regulation of secondary cell wall biosynthesis in Arabidopsis. Plant Cell Physiol. 50, 1950-1964. https://doi.org/10.1093/pcp/pcp139.

Medina-Puche, L., Cumplido-Laso, G., Amil-Ruiz, F., Hoffmann, T., Ring, L., Rodriguez-Franco, A., Caballero, J.L., Schwab, W., MunozBlanco, J., and Blanco-Portales, R. (2014). MYB10 plays a major role in the regulation of flavonoid/phenylpropanoid metabolism during ripening of Fragaria $\times$ ananassa fruits. J. Exp. Bot. 65, 401-417. https://doi.org/10.1093/jxb/ert377. 
Mitsuda, N., Seki, M., Shinozaki, K., and Ohme-Takagi, M. (2005). The NAC transcription factors NST1 and NST2 of Arabidopsis regulate secondary wall thickenings and are required for anther dehiscence. Plant Cell 17, 2993-3006. https://doi.org/10.1105/tpc.105.036004.

Mmadi, M.A., Dossa, K., Wang, L., Zhou, R., Wang, Y., Cisse, N., Sy, M.O., and Zhang, X. (2017). Functional characterization of the versatile MYB gene family uncovered their important roles in plant development and responses to drought and waterlogging in Sesame. Genes (Basel) 8, 362. https://doi.org/10.3390/genes8120362.

Nie, J., Cai, Y., Zhang, S., Lin, Y., Xu, Y., and Zhang, J. (2009). The anatomic study on relationship of stone cells and parenchyma cells during fruit development of Pyrus bretschneideri. Acta Hortic. Sin. 36(8), 12081214. https://doi.org/10.16420/j.issn.0513-353x.2009.08.019.

Riechmann, J.L., and Ratcliffe, O.J. (2000). A genomic perspective on plant transcription factors. Curr. Opin. Plant Biol. 3, 423-434. https://doi.org/10.1016/s1369-5266(00)00107-2.

Scully, E.D., Gries, T., Sarath, G., Palmer, N.A., Baird, L., Serapiglia, M.J., Dien, B.S., Boateng, A.A., Ge, Z., Funnell-Harris, D.L., Twigg, P., Clemente, T.E., and Sattler, S.E. (2016). Overexpression of SbMyb60 impacts phenylpropanoid biosynthesis and alters secondary cell wall composition in Sorghum bicolor. Plant J. 85, 378-395. https:// doi.org/10.1111/tpj.13112.

Smith, R.A., Schuetz, M., Roach, M., Mansfield, S.D., Ellis, B., and Samuels, L. (2013). Neighboring parenchyma cells contribute to Arabidopsis xylem lignification, while lignification of interfascicular fibers is cell autonomous. Plant Cell 25, 3988-3999. https://doi. org/10.1105/tpc.113.117176.

Tuan, P.A., Bai, S., Yaegaki, H., Tamura, T., Hihara, S., Moriguchi, T., and Oda, K. (2015). The crucial role of PpMYB10.1 in anthocyanin accumulation in peach and relationships between its allelic type and skin color phenotype. BMC Plant Biol. 15, 280. https://doi. org/10.1186/s12870-015-0664-5.

Wang, Y., Zhang, X., Yang, S., Wang, C., Lu, G., Wang, R., Yang, Y., and Li, D. (2017). Heterogenous expression of Pyrus pyrifolia PpCAD2 and $P p E X P 2$ in tobacco impacts lignin accumulation in transgenic plants. Gene 637, 181-189. https://doi.org/10.1016/j.gene.2017.09.056.

Wu, L.F., Hughes, T.R., Davierwala, A.P., Robinson, M.D., Stoughton, R., and Altschuler, S.J. (2002). Large-scale prediction of Saccharomyces cerevisiae gene function using overlapping transcriptional clusters. Nat. Genet. 31, 255-265. https://doi.org/10.1038/ng906.

Yan, C., Yin, M., Zhang, N., Jin, Q., Fang, Z., Lin, Y., and Cai, Y. (2014). Stone cell distribution and lignin structure in various pear varieties. Sci. Hortic. 174, 142-150. https://doi.org/10.1016/j. scienta.2014.05.018.

Yang, C., Xu, Z., Song, J., Conner, K., Vizcay Barrena, G., and Wilson, Z.A. (2007). Arabidopsis MYB26/MALE STERILE35 regulates secondary thickening in the endothecium and is essential for anther dehiscence. Plant Cell 19, 534-548. https://doi.org/10.1105/tpc.106.046391.

Yang, J.H., and Wang, H. (2016). Molecular mechanisms for vascular development and secondary cell wall formation. Front Plant Sci. 7, 356. https://doi.org/10.3389/fpls.2016.00356.

Zhang, C., Ma, R., Xu, J., Yan, J., Guo, L., Song, J., Feng, R., and Yu, M. (2018). Genome-wide identification and classification of MYB superfamily genes in peach. PLoS ONE 13, e0199192. https://doi. org/10.1371/journal.pone.0199192.

Zhao, P., Li, Q., Li, J., Wang, L., and Ren, Z. (2014). Genome-wide identification and characterization of $R 2 R 3 M Y B$ family in Solanum lycopersicum. Mol. Genet. Genomics 289, 1183-1207. https://doi. org/10.1007/s00438-014-0879-4.

Zhong, R., and Ye, Z.H. (2012). MYB46 and MYB83 bind to the SMRE sites and directly activate a suite of transcription factors and secondary wall biosynthetic genes. Plant Cell Physiol. 53, 368-380. https://doi.org/10.1093/pcp/pcr185.
Zhong, R., and Ye, Z.H. (2015). Secondary cell walls: biosynthesis, patterned deposition and transcriptional regulation. Plant Cell Physiol. 56, 195-214. https://doi.org/10.1093/pcp/pcu140.

Zhong, R., Richardson, E.A., and Ye, Z.H. (2007). The MYB46 transcription factor is a direct target of SND1 and regulates secondary wall biosynthesis in Arabidopsis. Plant Cell 19, 2776-2792. https:// doi.org/10.1105/tpc.107.053678.

Zhong, R., Lee, C., Zhou, J., McCarthy, R.L., and Ye,Z.H. (2008). A battery of transcription factors involved in the regulation of secondary cell wall biosynthesis in Arabidopsis. Plant Cell 20, 2763-2782. https:// doi.org/10.1105/tpc.108.061325.

Zhong, R., Lee, C., and Ye, Z.H. (2010). Evolutionary conservation of the transcriptional network regulating secondary cell wall biosynthesis. Trends Plant Sci. 15, 625-632. https://doi. org/10.1016/j.tplants.2010.08.007.

Zhong, R., Lee, C., McCarthy, R.L., Reeves, C.K., Jones, E.G., and Ye, Z.H. (2011). Transcriptional activation of secondary wall biosynthesis by rice and maize NAC and MYB transcription factors. Plant Cell Physiol. 52, 1856-1871. https://doi.org/10.1093/pcp/pcr123.

Zhou, J., Lee, C., Zhong, R., and Ye, Z.H. (2009). MYB58 and MYB63 are transcriptional activators of the lignin biosynthetic pathway during secondary cell wall formation in Arabidopsis. Plant Cell 21, 248-266. https://doi.org/10.1105/tpc.108.063321.

Received: Dec. 14, 2020

Accepted: Feb. 27, 2021

Addresses of authors:

Kuerban Tusong ${ }^{1}$, Aisajan Mamat ${ }^{1, *}$, Hongmei $\mathrm{Mu}^{2}$ and Juan $\mathrm{Xu}^{1}$

${ }^{1}$ Institute of Horticultural Crops, Xinjiang Academy of Agricultural Sciences, 403 Nanchang Road,

Urumqi 830091, China

2 Yantai Academy of Agricultural Sciences, Yantai 265500, Shandong, China

\section{SUPPLEMENTAL INFORMATION}

For Supplemental Information see www.ishs.org/eJHS. 